\title{
Prognostic Factors of Response to Laparoscopic Splenectomy in Patients with Idiopathic Thrombocytopenic Purpura
}

\begin{abstract}
Laparoscopic splenectomy (LS) has become the treatment of choice for patients with idiopathic thrombocytopenic purpura (ITP) who do not respond to medical treatment. The aim of this study was to identify factors predictive of outcome after LS for ITP. From May 1997 to December 2002, we performed 30 LS on patients with ITP. A positive response was defined as a postoperative platelet count greater than $50,000 / \mu \mathrm{L}$ and no requirement for maintenance therapy. Chi-square testing was performed to determine the predictive effects of the following variables: age, sex, preoperative response to steroids or immunoglobulin, duration of disease, antiplatelet antibody, platelet associated antibody, and antinuclear antibody. LS was successfully performed in all patients. For a mean follow-up interval of 24.3 months, response to LS was $73.3 \%$. Splenectomy for steroid nonresponders resulted in an inferior complete response rate (10 of 18,55.6\%) as compared with those that experienced relapse after steroid treatment ( 11 of $12,91.7 \%)(p=0.042)$. The other significant predictor of outcome by univariate analysis was the time between diagnosis and surgery $(p=0.049)$. The other variables showed no significant correlation with successful splenectomy. We conclude that LS can be performed safely with a satisfactory remission rate in patients with ITP who do not respond to medical treatment, and that the factors most frequently associated with surgical success are a response to steroid and disease duration.
\end{abstract}

Key Words : Purpura, Thrombocytopenic, Idiopathic; Laparoscopy; Splenectomy

\author{
Hyuk-Chan Kwon, Chang Hoon Moon, \\ Young Rak Cho, Min Chan Kim*, \\ Kyeong Hee Kim ${ }^{\dagger}$, Jin Yeong Han', \\ Young Ho Lee, Sung Yong Oh, \\ Sung-Hyun Kim, Jae-Seok Kim, \\ Hyo-Jin Kim
}

Departments of Internal Medicine, Surgery* Laboratory Medicine ${ }^{\dagger}$, Pediatrics ${ }^{\ddagger}$, College of Medicine, Dong-A University, Busan, Korea

Received : 27 October 2004

Accepted : 24 January 2005

\section{Address for correspondence}

Hyo-Jin Kim, M.D.

Department of Internal Medicine, Dong-A University,

1-3 ga Dongdaeshin-dong, Seo-gu, Busan 602-715, Korea

Tel : +82.51-240-2951, Fax : +82.51-240-2088

E-mail : kimhi@mail.donga.ac.kr

\section{INTRODUCTION}

Immune thrombocytopenic purpura (ITP) is an autoimmune disorder characterized by accelerated platelet destruction (1). The spleen is the primary site of platelet destruction and antiplatelet antibody production, and the mainstay medical therapy is bolus corticosteroids followed by a tapering dose. However, long-term remission rates are only $20 \%$ to $25 \%$ in adults, and for patients who continue to require steroids or who fail to respond to high-dose corticosteroids, splenectomy is considered a second-line therapy (2).

Laparoscopic splenectomy (LS) was first reported in 1992 (3), and is now used to treat a number of hematologic diseases, including ITP. Several reports that have compared the results of open splenectomy and LS for ITP have concluded that LS results in fewer postoperative complications $(4,5)$. However, no difference was found in remission rates. LS has the following advantages; reduced operative trauma, less splenosis, better detection of accessory spleens, less post-operative pain, cosmetic advantages, early discharge (mostly within $48 \mathrm{hr}$ after surgery), and it allows patients to resume normal activity within a few days (6-9). However, prognostic indicators are important because for patients with an expected good response to LS the benefits of the procedure would justify the associated risks. However, previous attempts to identify factors that predict LS success have led to conflicting results (10-13).

Here we report on the intermediate follow-up results of 30 patients who underwent LS because of nonresponsive or refractory ITP. We also retrospectively analyzed preoperative factors to identify those predictive of a successful outcome following LS.

\section{MATERIALS AND METHODS}

\section{Patients}

From May 1997 to December 2002, 30 ITP patients who had not responded to medical treatment underwent LS at the Department of Surgery at Dong-A University Medical Center, Busan, Korea. ITP was diagnosed according to the guidelines of the American Society of Hematology (2). All patients underwent bone marrow aspiration and other causes of thrombocytopenia were ruled out.

All 30 patients had been treated with prednisone at an initial dose of $1 \mathrm{mg} / \mathrm{kg} /$ day for one month, which was then 
tapered to the minimal effective dose required to maintain platelets at $>30,000 / \mu \mathrm{L}$. Those who were refractory to steroid therapy, relapsed during steroid tapering, intolerant to medical treatment, pregnant, and those actively bleeding with platelet counts $<30,000 / \mu \mathrm{L}$ were selected for splenectomy. The patients were administered a polyvalent pneumococcal vaccine at least 2 weeks before surgery, and $400 \mathrm{mg}$ of intravenous immunoglobulin G (IVIG) per kilogram of body weight for five consecutive days immediately prior to laparoscopic splenectomy.

\section{Operation technique}

The detailed operative procedure has been described (14). Briefly, patients were placed in a right lateral position, and the procedure was performed through $5-10 \mathrm{~mm}$ incisions. Abdomens were insufflated with $\mathrm{CO}_{2}$, and three laparoscopic ports were introduced to allow a laparoscope and surgical instruments access to the abdominal cavity. In each case, the spleen was mobilized by dividing peritoneal attachments and ligaments by electrocautery. The left upper quadrant, splenocolic ligament, splenic hilum, lesser sac, root of the small bowel mesentery, and pelvis were routinely explored, and accessory spleens were removed. Spleens were placed in a plastic bag, the opening of which was delivered out through the most lateral port. Specimen were mechanically fracture-morselized via the exteriorized extraction bag. After withdrawing the spleen, the operative field was irrigated with normal saline, and the possibility of bleeding or of a missed accessory spleen investigated.

\section{Clinical outcome and follow-up}

In this study we adopted the definition of hematologic outcome suggested by Radaelli et al. (15). Complete response (CR) was defined as a rise in the platelet count to $>100,000 / \mu \mathrm{L}$ for at least 6 months (with or without treatment), partial response (PR) was defined as a platelet count of 50,000-100,000/ $\mu \mathrm{L}$, and refractory disease (no response, NR) was defined no platelet count increase postoperatively or a platelet count of $<50,000 / \mu \mathrm{L}$ during treatment. For the statistical analysis patients were subdivided into two groups, namely, responder $(\mathrm{CR}+\mathrm{PR})$ and non-responder (NR) groups. We checked the platelet count at 1,2, 4, and 8 weeks, and thereafter we followed the patients at 3-month intervals for $1 \mathrm{yr}$, and then annually. Immediate response to splenectomy was determined within 6 months of surgery and the long-term response from 6 months to 7 yr after surgery.

\section{Prognostic factors of laparoscopic splenectomy outcome}

Patient age, sex, prior response to steroids, response to IVIG, time from diagnosis to splenectomy, and positivity to antiplatelet antibody, platelet associated antibody, and antinu- clear antibody (ANA) were evaluated to identify the characteristics of responders to LS. Categorical and numerical data were evaluated using the chi-square and Mann-Whitney tests, and $p$ value of $<0.05$ was considered statistically significant.

\section{RESULTS}

\section{Patient's characteristics and the outcome of splenectomy}

The mean age of the 30 patients enrolled in this study was $28 \mathrm{yr}$ (range, 3-71). There were 7 males (23\%) and 23 females $(67 \%)$, and 7 were pediatric patients. The mean time from diagnosis to splenectomy was 7.5 months (range, 1-88.8). Surgical indications included disease refractory to medical treatment $(18 / 30,60 \%)$, or recurrence during tapering (12/30, $40 \%)$.

All 30 patients successfully underwent LS without the need for conversion to open splenectomy. There were no perioperative deaths. Three patients $(10 \%)$ experienced transient postoperative complications, namely, infections, subphrenic abscesses, and minor wound hematomas, respectively. The mean operative time for LS was 117.5 min (range, 80-360), and the mean postoperative hospital stay was 4 days (range, 2-15). Accessory spleens were found and removed in five patients (16.7\%), and two of these patients had two accessory spleens. The locations of the accessory spleen were; near the hilum, and on the greater omentum and splenocolic ligament.

At a mean follow-up of 24.3 months, the initial response rate was $73.3 \%$ (CR in 15/30 [50\%], and PR in 7/30 [23.3\%]). All eight non-responders underwent radionucleotide spleen scans, and one of these eight was found to have an accessory spleen, which was successfully removed laparoscopically. In this patient, the thrombocytopenia recurred 12 months after surgery. The platelet count rose following the removal of the accessory spleens, and this patient has been in remission for 14 months. Four patients had never been administered postoperative medication for ITP because they were asymptomatic and had platelet counts between 30,000 and 50,000/ $\mu \mathrm{L}$. Three patients were treated with high dose dexamathasone (40 mg intravenously for 4 days), however no definite response was observed, and these patients continue to take danazol and/or vincristine intermittently to maintain platelet count levels. One patient with an initial response to LS subsequently developed recurrent disease which required no treatment. Therefore, the overall remission rate was $73.3 \%$ (22 of 30).

\section{Prognostic factors of response}

The relationship between the investigated variables and LS remission rates for ITP are listed in Table 1. Preoperative responsiveness to steroid therapy was found to be significantly correlated with response to LS. Moreover, splenectomy for 
Table 1. Patients' characteristics related to the response to laparoscopic splenectomy

\begin{tabular}{|c|c|c|c|}
\hline Variables & Responder & Non-responder & $p$ \\
\hline \multicolumn{4}{|l|}{ Age } \\
\hline$<15$ yr $(n=7)$ & $6(85.7 \%)$ & $1(14.3 \%)$ & \multirow[t]{2}{*}{0.393} \\
\hline$\geq 15$ yr $(n=23)$ & $15(65.2 \%)$ & $8(34.8 \%)$ & \\
\hline \multicolumn{4}{|l|}{ Sex } \\
\hline Male $(n=7)$ & $4(57.1 \%)$ & $3(42.9 \%)$ & \multirow[t]{2}{*}{1.000} \\
\hline Female $(n=23)$ & $17(73.9 \%)$ & $6(26.1 \%)$ & \\
\hline \multicolumn{4}{|l|}{ Disease duration } \\
\hline$<12$ months $(n=17)$ & $9(52.9 \%)$ & $8(47.1 \%)$ & \multirow[t]{2}{*}{0.042} \\
\hline$\geq 12$ months $(n=13)$ & $12(92.3 \%)$ & $1(7.7 \%)$ & \\
\hline \multicolumn{4}{|l|}{ Response to steroid } \\
\hline$+(n=12)$ & $11(91.7 \%)$ & $1(8.3 \%)$ & \multirow[t]{2}{*}{0.049} \\
\hline$-(n=18)$ & $10(55.6 \%)$ & $8(44.4 \%)$ & \\
\hline \multicolumn{4}{|l|}{ Response to IVIG* } \\
\hline$+(n=18)$ & $13(72.2 \%)$ & $5(27.8 \%)$ & \multirow[t]{2}{*}{1.000} \\
\hline$-(n=12)$ & $8(66.7 \%)$ & $4(33.3 \%)$ & \\
\hline \multicolumn{4}{|l|}{ Anti-platelet antibody } \\
\hline$+(n=7)$ & $5(71.4 \%)$ & $2(28.6 \%)$ & \multirow[t]{2}{*}{1.000} \\
\hline$-(n=18)$ & $11(61.1 \%)$ & $7(38.9 \%)$ & \\
\hline \multicolumn{4}{|c|}{ Platelet associated antibody } \\
\hline$+(n=13)$ & $7(53.8 \%)$ & $6(46.2 \%)$ & \multirow[t]{2}{*}{0.411} \\
\hline$-(n=12)$ & $9(75.0 \%)$ & $3(25.0 \%)$ & \\
\hline \multicolumn{4}{|l|}{ ANA $^{\dagger}$} \\
\hline$+(n=7)$ & $4(57.1 \%)$ & $3(42.9 \%)$ & \multirow[t]{2}{*}{1.000} \\
\hline$-(n=13)$ & $8(61.5 \%)$ & $5(38.5 \%)$ & \\
\hline
\end{tabular}

IVIG, intravenous immunoglobulin G; ANA, antinuclear antibody.

steroid nonresponders resulted in an poorer CR rate (10 of $18,55.6 \%)$ than for those who relapsed after steroid treatment (11 of $12,91.7 \%)(p=0.042)$. The other significant predictor of a satisfactory outcome by univariable analysis was the time between diagnosis and surgery. Patients with a disease duration $>12$ months had a higher response than those with a duration less than this $(92.3 \%$ vs. $52.9 \%$, respectively) $(p=0.049)$, and pediatric patients responded better (6/7, $85.7 \%)$ than adults $(16 / 23,69.6 \%)$, but without significance $(p=0.393)$. When we analyzed the data after excluding pediatric patients, no difference in response rate was found between those older and younger than $40 \mathrm{yr}$ old $(71.4 \%$ vs. $62.5 \%$, respectively, $p=1.000$ ). The mean operative time for responders was $129 \pm 59 \mathrm{~min}$, and for non-responder $149 \pm 53 \mathrm{~min}$ $(p=0.385)$. Not all patients received serologic tests for antiplatelet antibody, platelet associated antibody, and ANA, but the test results obtained showed no relation with $\mathrm{LS}$ response. And, the other variables examined were not found to be predictive of successful splenectomy.

\section{DISCUSSION}

The recent emergence of minimally invasive techniques has increased interest in splenectomy for a variety of hematologic illnesses, including ITP. The response rate of LS for ITP is reported to be $64 \%$ to $90 \%$ (6-13); however, it is dif- ficult to compare study results. Most LS reports are retrospective in nature, involve data collections over protracted periods of time, and have failed to follow patients systematically and prospectively. Furthermore, there does not appear to be any standard definition of disease remission and follow-up duration, and whereas some authors define disease remission as the normalization of platelet count, other have defined it to be a normalization of platelet count without the need for future medical therapy. In the present study, we defined disease remission as a sustained platelet count of $>50,000 / \mu \mathrm{L}$ without the need for medical therapy. We obtained an overall remission rate of $73.3 \%$ at a median follow-up of 24.3 months, which concurs with other reports (6-13).

The natural history of ITP differs in adults and children. Spontaneous remission, independent of treatment, occurs in $>80 \%$ of children with the disease, and when necessary, splenectomy is nearly always curative in children $(16,17)$. The guidelines issued by the American Society of Hematology recommend that splenectomy be considered for children who have had ITP for at least one year with symptomatic severe thrombocytopenia (2). In the present study, the enrolled pediatric patients had an ITP duration of $>12$ months and were refractory to steroid or IVIG, and most responded well after $\operatorname{LS}(6 / 7,85.7 \%)$.

The overlooking of accessory spleens is a major cause of LS failure in ITP. In the present study the accessory spleen rate was $16.7 \%$, which is comparable to the rates $(5-21 \%)$ reported for ITP patients that have undergone LS $(9,12)$. One of our patients with postsurgical ITP recurrence was found to have an accessory spleen, which was removed successfully by using a laparoscopic procedure.

Many investigators have sought to identify the preoperative variables that predict a successful outcome following LS; however, the results obtained were often divergent (9-13). In the present study we considered some of the parameters previously examined in the literature, such as, age, sex, response to initial steroid treatment and/or IVIG therapy, time between diagnosis and surgery, and positivity for anti-platelet antibody, platelet associated antibody, and ANA. However, we did not evaluate platelet kinetics or platelet sequestration because the guidelines published by the American Society of Hematology deems them unnecessary (2).

Our results show that an initial response to steroid treatment is significantly associated with a positive response to LS. This correlation has been previously reported (9), although a more recent study failed to identify a significant association (12). In the present study, the other significant prognostic factor of successful LS identified was the time from diagnosis to LS. Specifically, patients who received LS $>12$ months after diagnosis had a better response. This finding may be due to most patients with previous responded to steroid therapy (10 out of 12 patients) and all of the pediatric patients had time between diagnosis and LS was more than 12 months. It is not similar with other results (18). 
Patient age has most often been identified as a predictor of a successful response to LS (9-12, 19). Several studies have shown that an age between 30 and 45 years is an significant prognostic indicator of successful LS $(10,11,19)$. However, other studies have produced different results $(9,12)$. The predictive strength of preoperative response to IVIG is also controversial. One study demonstrated a significant correlation between response to IVIG and sustained response to splenectomy (18), whereas other studies disagree $(11,15)$. These discrepancies between studies are probably due to the different ages of the populations studied and the varying lengths of follow-up.

Recently, the long-term outcomes of a group of adult chronic ITP patients after splenectomy failure were reported. It was found that most patients with refractory ITP eventually achieved stable remission, but it was emphasized that this occurred slowly (20). In the present study, $50 \%$ of the patients had a platelet count of 30,000-50,000/ $\mu \mathrm{L}$ after LS, and these patients did not require medication. And, although it has been reported that refractory ITP patients may respond to high dose dexamethosone therapy (21), in the present study we did not observe same result. In conclusion, our results indicated that an initial response to steroid and a period of more than 12 months between diagnosis and LS are positive predictors of long-term remission in ITP.

\section{REFERENCES}

1. Cines DB, Blanchette VS. Immune thrombocytopenic purpura. $N$ Engl J Med 2002; 346: 995-1008.

2. George JN, Woolf SH, Raskob GE, Wasser JS, Aledort LM, Ballem PJ, Blanchette VS, Bussel JB, Cines DB, Kelton JG, Lichtin AE, McMillan R, Okerbloom JA, Regan DH, Warrier I. Idiopathic thrombocytopenic purpura: a practice guideline developed by the explicit methods for the American Society of Hematology. Blood 1996; 88: $3-40$.

3. Delaitre B, Maignien B. Laparoscopic splenectomy-technical aspects. Surg Endosc 1992; 6: 305-8.

4. Brunt LM, Langer JC, Quasebarth MA, Whitman ED. Comparative analysis of laparoscopic versus open splenectomy. Am J Surg 1996; 172: 596-601.

5. Watson DI, Coventry BJ, Chin T, Gill PG, Malycha P. Laparoscopic versus open splenectomy for immune thrombocytopenic purpura. Surgery 1997; 121: 18-22.

6. Szold A, Schwartz J, Abu-Abeid S, Bulvik S, Eldor A. Laparoscopic splenectomies for idiopathic thrombocytopenic purpura: experience of sixty cases. Am J Hematol 2000; 63: 7-10.
7. Pace DE, Chiasson PM, Schlachta CM, Mammazza J, Poulin EC. Laparoscopic splenectomy for idiopathic thrombocytopenic purpura (ITP) Long term follow-up data. Surg Endosc 2003; 17: 95-8.

8. Kang JK, Lee WJ, Kim KS, Kim BR. Laparoscopic splenectomy for idiopathic thrombocytopenic purpura. J Korean Surg Soc 1996; 50: 846-51.

9. Stanton CJ. Laparoscopic splenectomy for idiopathic thrombocytopenic purpura (ITP). A five-year experience. Surg Endosc 1999; 13: 1083-6.

10. Katkhouda N, Grant SW, Mavor E, Friedlander MH, Lord RV, Achanta K, Essani R, Mason R. Predictors of response after laparoscopic splenectomy for immune thrombocytopenic purpura. Surg Endosc 2001; 15: 484-8.

11. Duperier T, Brody F, Felsher J, Walsh RM, Rosen M, Ponsky J. Predictive factors for successful laparoscopic splenectomy in patients with immune thrombocytopenic purpura. Arch Surg 2004; 139: 61-6.

12. Wu JM, Lai IR, Yuan RH, Yu SC. Laparoscopic splenectomy for idiopathic thrombocytopenic purpura. Am J Surg 2004; 187: 720-3.

13. Schwartz J, Leber MD, Gillis S, Giunta A, Eldor A, Bussel JB. Long term follow-up after splenectomy performed for immune thrombocytopenic purpura (ITP). Am J Hematol 2003; 72: 94-8.

14. Lee WJ, Kim BR. Laparoscopic splenectomy for chronic idiopathic thrombocytopenic purpura. Surg Laparosc Endosc 1997; 7: 209-12.

15. Radaelli F, Faccini P, Goldaniga M, Guggiari E, Pozzoli E, Maiolo AT, Ciani A, Pogliani EM. Factors predicting response to splenectomy in adult patients with idiopathic thrombocytopenic purpura. Haematologica 2000; 85: 1040-4.

16. Davis PW, Williams DA, Shamberger RC. Immune thrombocytopenia: surgical therapy and predictors of response. J Pediatr Surg 1991; 26: 407-12.

17. Jacir NN, Robertson FM, Crombleholme TM, Harris BH. Recurrence of immune thrombocytopenic purpura after splenectomy. $J$ Pediatr Surg 1996; 31: 115-6.

18. Choi CW, Kim BS, Seo JH, Shin SW, Kim YH, Kim JS, Sohn SK, Kim JS, Shin DG, Ryoo HM, Lee KH, Lee JJ, Chung IJ, Kim HJ, Kwak JY, Yim CY, Ahn JS, Lee JA, Park YS. Response to highdose intravenous immune globulin as a valuable factor predicting the effect of splenectomy in chronic idiopathic thrombocytopenic purpura patients. Am J Hematol 2001; 66: 197-202.

19. Fabris F, Tassan T, Ramon R, Carraro G, Randi ML, Luzzatto G, Moschino P, Girolami A. Age as the major predictive factor of longterm response to splenectomy in immune thrombocytopenic purpura. Br J Haematol 2001; 112: 637-40.

20. McMillan R, Durette C. Long-term outcomes in adults with chronic ITP after splenectomy failure. Blood 2004; 104: 956-60.

21. Andersen JC. Response of resistant idiopathic thrombocytopenic purpura to pulsed high-dose dexamethasone therapy. $N$ Engl J Med 1994; 330: 1560-4. 